\title{
Zintl phases for thermoelectric devices
}

\author{
Susan M. Kauzlarich, ${ }^{* a}$ Shawna R. Brown ${ }^{a}$ and G. Jeffrey Snyder ${ }^{b}$ \\ Received 13th February 2007, Accepted 11th April 2007 \\ First published as an Advance Article on the web 24th April 2007 \\ DOI: $10.1039 /$ b702266b
}

By converting waste heat into electricity and improving the efficiency of refrigeration systems, thermoelectric devices could play a significant role in solving today's energy problems. Increasing the thermoelectric efficiency (as measured by the thermoelectric material's figure-of-merit, $z T$ ) is critical to the development of this technology. Complex Zintl phases, in particular, make ideal candidates for thermoelectric materials because the necessary "electron-crystal, phonon-glass" properties can be engineered with an understanding of the Zintl chemistry. A recent example is the discovery that $\mathrm{Yb}_{14} \mathrm{MnSb}_{11}$, a transition metal Zintl compound, has twice the $z T$ as the material currently in use at NASA. This perspective outlines a strategy to discover new high $z T$ materials in Zintl phases, and presents results pointing towards the success of this approach.

\section{Introduction}

In recent years there has been a renewed effort to identify high performance thermoelectric materials for applications in power generation and device cooling. Several factors have driven this interest, including environmental concern with the use of chlorofluorocarbons in compressor-based refrigeration and the potential to generate electrical power from low-level waste heat. In particular, thermoelectric power generation is being considered for use in automobiles; here, the waste exhaust heat can be utilized, thereby improving the overall fuel economy of the vehicle. Fig. 1 shows a schematic of a thermoelectric device. Heat from above drives the free carriers (electrons in the n-type leg, holes in the ptype leg) from the hot side of the thermoelectric material to the cold side, thereby creating electrical power in the load. Alternatively, thermoelectrics can be used for refrigeration because replacing the load with a voltage source will force heat to flow from the top side to the bottom side. Because they have no moving parts and use no liquid refrigerant, thermoelectric refrigerators and power generators are particularly well-suited to applications in which long-term, unattended operation is necessary, or very small devices are to be cooled. ${ }^{1}$ Current applications include power generation for deep-space probes or remote sensors, and thermal management of high power electronics. While many applications of thermoelectric devices can be envisioned, their widespread implementation is limited as a result of the low efficiency that today's thermoelectric materials afford.

The efficiency of a thermoelectric device generally improves by increasing the material's thermoelectric figure of merit $z T=$ $\alpha^{2} T / \rho \kappa\left(\alpha\right.$ : Seebeck coefficient $\left(\mathrm{V} \mathrm{K}^{-1}\right), T$ : temperature $(\mathrm{K}), \rho$ : electrical resistivity $(\Omega \mathrm{cm}), \kappa$ : thermal conductivity $\left(\mathrm{W} \mathrm{cm}^{-1} \mathrm{~K}^{-1}\right){ }^{1,2}$ For metals or degenerate semiconductors the Seebeck coefficient is proportional to temperature, effective mass $m^{*}$, and inversely proportional to the charge carrier concentration $n$. In the approximation of energy independent scattering, the relationship is

${ }^{a}$ Department of Chemistry, University of California, One Shields Ave, Davis, CA, 95616, USA

${ }^{b}$ California Institute of Technology, 1200 California Blvd., Pasadena, CA, 91125, USA

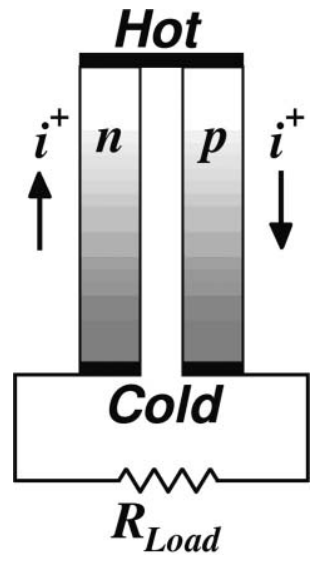

Fig. 1 Schematic of a thermoelectric generator.

given $^{3}$ by

$$
a=\frac{8 \pi^{2} k_{\mathrm{B}}^{2}}{3 e h^{2}} m^{*} T\left(\frac{\pi}{3 n}\right)^{2 / 3}
$$

where $k_{\mathrm{B}}$ is Boltzmann's constant, $e$ is the charge of an electron and $h$ is Planck's constant. Thus, large thermopower $|a|$ translates to large effective mass which is equivalent to large density-of-states or flat bands at the Fermi level. Low electrical resistivity (or high electrical conductivity $\sigma$ ) requires a large mobility, $\mu$, and large carrier (electrons or holes) concentration $n$ through

$$
1 / \rho=\sigma=n e \mu
$$

High $z T$ requires a combination of the large thermopower found in insulators or semiconductors with the low electrical resistivity of metals. This is typically only achieved in heavily doped semiconductors combining high electron density-of-states (high effective masses) with high carrier concentrations, and high mobility ${ }^{1}$ as seen in eqn (1) and (2) for $\alpha$ and $\sigma$.

The thermal conductivity $\kappa$ can be separated into two parts: the lattice or phonon contribution $\kappa_{1}$ and the electronic contribution $\kappa_{\mathrm{e}}$, which is proportional to the electrical conductivity $\kappa_{\mathrm{e}}=L \sigma T$ (with the Lorenz number $L=\pi^{2} k_{\mathrm{B}}{ }^{2} / 3 e^{2}$ ). Thus in addition to optimizing the electronic transport properties, the lattice thermal 
Susan M. Kauzlarich obtained her B. S. in 1980 from the College of William and Mary, and her Ph.D. in 1985 from Michigan State University working with Professor Bruce Averill. She was a postdoctoral fellow with Professor John D. Corbett at Iowa State University $1985-1987$. She started as an Assistant Professor at the University of California, Davis in 1987 and is currently a full Professor at the same institution. She took a sabbatical at Argonne National Laboratory (ANL) in 1992 and held a visiting Scholar Appointment at ANL 1997-1998. She is the recipient of the Maria Goeppert Mayer Distinguished Scholar Award, ANL 1997. She has received awards for mentoring, including the Outstanding Mentoring Award in 2001 and the UC Davis Distinguished Graduate Mentoring Award in 2004. She has published over 160 papers in the field of solid state and materials chemistry and is the author of 1 patent. She has edited one book entitled Chemistry, Structure and Bonding of Zintl Phases and Ions, published in 1991 and a MRS Symposium Proceedings on Solid State Chemistry of Inorganic Materials (1999). She is currently on the Advisory Review Board for Research Corporation (2004-present) and the Editorial Advisory Board for the Handbook on the Physics and Chemistry of Rare Earths (2002-present). She has been on numerous editorial advisory boards for journals including Inorganic Chemistry, Chemistry of Materials, and Journal of Solid State Chemistry. She was the Associate Editor for the Journal of Solid State Chemistry (2000-2005). She is currently an Editor for Chemistry of Materials (2006-present). Her current research is focused on the synthesis, characterization of transition metal Zintl phases for thermoelectric and magnetic applications. She discovered the first transition metal Zintl phase, $\mathrm{Ca}_{14} \mathrm{MnBi}_{11}$ and went on to discover colossal magnetoresistance and efficient thermoelectric properties in compounds of this structure. She has demonstrated the synthetic design and control of silicon nanoparticles with applications for hydrogen storage and as light emitting quantum dots. She has also published in the area of Fe/Au core/shell nanoparticle synthesis.

Shawna R. Brown received her Bachelor's of Science in chemistry in 2001 from the University of California, Davis. She continued her education at UC Davis and achieved a Master's degree in inorganic chemistry in 2005 while working under the supervision of Professor Susan M. Kauzlarich. Her thesis titled, Thermoelectric Investigation of $\mathrm{Yb}_{14} \mathrm{MSb}_{11}(\mathrm{M}=\mathrm{Mn}, \mathrm{Zn})$ and $\mathrm{A}_{11} \mathrm{Sb}_{10}(\mathrm{~A}=\mathrm{Ca}, \mathrm{Yb})$, focuses on examining the thermoelectric properties of Zintl compounds. Shawna's graduate research unearthed a novel thermoelectric material $\left(\mathrm{Yb}_{14} \mathrm{MnS} b_{11}\right)$ for which she was recognized by the National Aeronautics and Space Administration. As a result of the exceptional properties of $\mathrm{Yb}_{14} \mathrm{MnSb}_{11}$ and related compounds, she has formed a start-up company, High Merit Thermoelectrics, Inc., that specializes in thermoelectric materials and devices. To date, her company has secured patenting and licensing rights for $\mathrm{Yb}_{14} \mathrm{MnSb_{11 }}$, and is currently seeking funding to initiate their prototyping stage. In the future she aims to continue to develop improved thermoelectric materials for power generation applications.

G. Jeffrey Snyder obtained his B.S. degree in physics, chemistry and mathematics at Cornell University (1991) focusing on solid state chemistry of chalcogenides under the direction of Professor Frank DiSalvo. During 1992-1993 he studied the solid state chemistry of intermetallics, sub-nitrides and $C_{60}$ materials with Professor Arndt Simon at the Max Planck Institut für Festkörperrperforschung in Stuttgart, Germany. He received his Ph.D. in applied physics from Stanford University (1997) where he was a Hertz Fellow. At Stanford he studied the magnetic and magneto-electrical transport properties of metallic perovskites under the direction of Professor Ted Geballe and Professor Mac Beasley. He was a Senior Member of the Technical Staff in the thermoelectrics group at NASA's Jet Propulsion Laboratory for 9 years (1997-2006) where he focused on thermoelectric materials and devices. Here he developed the thermoelectric compatibility concept for efficient segmentation of thermoelectric materials, electrochemical MEMS fabrication of thermoelectric microdevices, and a number of new, high efficiency thermoelectric materials. He is currently a Faculty Associate in materials science at the California Institute of Technology (Caltech). His recent interests include the discovery of new Zintl phase thermoelectric materials and nanostructured thermoelectric composites using bulk processing. Dr Snyder has published over 80 articles, and a number of patents including a recent chapter in the CRC Handbook of Thermoelectrics.

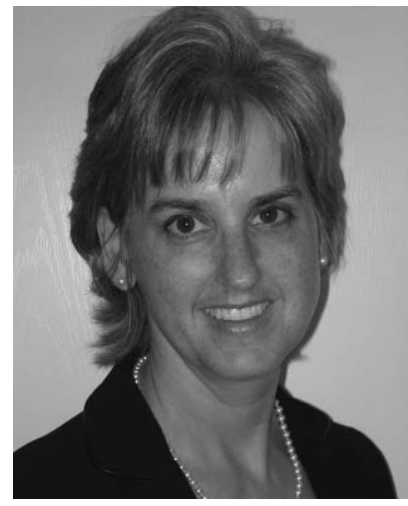

Susan M. Kauzlarich

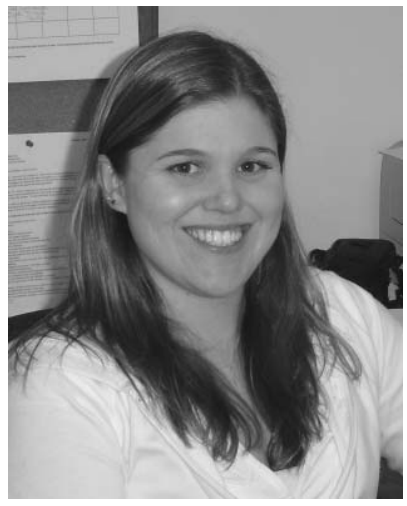

Shawna R. Brown

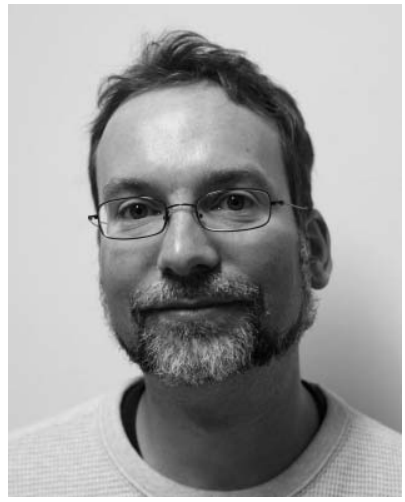

G. Jeffrey Snyder conductivity must also be low, such as that of an amorphous glass, to achieve high $z T$. These criteria have lead to the concept ${ }^{2}$ that the ideal material has the electronic structure of a compound semiconductor with the atomic structure of a glass, or an "electron- crystal, phonon-glass". Finally because $z T$ is very sensitive to carrier concentration (via $\alpha$ and $\rho$ ), it is essential to be able to control the carrier concentration through precise doping without disrupting the carrier mobility. 
Today's commercial thermoelectric materials are alloys discovered decades ago. The alloying of isoelectronic species (such as $\mathrm{Si}-\mathrm{Ge}$ alloys or $\mathrm{Bi}_{2} \mathrm{Te}_{3}-\mathrm{Sb}_{2} \mathrm{Te}_{3}-\mathrm{Bi}_{2} \mathrm{Se}_{3}$ alloys) introduces disorder which scatters phonons without too much disruption of the electron transport. While alloying substantially reduces lattice thermal conductivity, other mechanisms for reducing thermal conductivity are possible. Recent discoveries of high $z T$ materials in oxides, ${ }^{4}$ nanostructured materials, ${ }^{5,6}$ skutterudites, clathrates, chalcogenides, ${ }^{7}$ and Zintl phases ${ }^{8}$ indicate that there are many fruitful systems yet to consider.

For room-temperature applications, such as refrigeration and waste heat recovery up to $475 \mathrm{~K}, \mathrm{Bi}_{2} \mathrm{Te}_{3}$ and its alloys have proven to possess the greatest figure of merit for both $\mathrm{n}$ - and p-type thermoelectric systems. $\mathrm{Bi}_{2} \mathrm{Te}_{3}$ was first investigated as being a material of great thermoelectric promise in $1954 .{ }^{9}$ Today, more than five decades later, this compound semiconductor has remained the best commercial material for use near room temperature with maximum reported $z T$ typically in the range of 0.8 to 1.1 for optimized systems. ${ }^{1}$

For higher temperature power generation, materials based on Group 14 tellurides are typically employed such as PbTe, GeTe or SnTe. Reported $z T$ 's are usually less than 0.8. However, alloys, particularly with $\mathrm{AgSbTe}_{2}$, have lead to several reports of $z T>1$ for both n-type and p-type materials. ${ }^{10-13}$ The p-type alloy $(\mathrm{GeTe})_{0.85}\left(\mathrm{AgSbTe}_{2}\right)_{0.15}$ (TAGS) with maximum $z T$ greater than 1.2 has been successfully used in long-life thermoelectric generators.

\section{Zintl phases}

The combination of requirements inherent in $z T$ includes thermodynamic as well as both electrical and thermal transport properties (all of which can vary by orders of magnitude) which makes designing a thermoelectric material quite complex. One method to achieve this unusual mix of properties is to envision a complex material with distinct components providing different functions. This ideal thermoelectric material would have regions of the structure composed of a high mobility compound semiconductor that provides the "electron-crystal" electronic structure, interwoven (on a $\AA$ or nm scale) with a phonon inhibiting structure to act as the "phonon-glass". ${ }^{2}$ The phonon-glass region would benefit from disorder and therefore would be ideal to house dopants without disrupting the electron-crystal region.

Zintl phases are prime candidates for applying this concept to obtain high $z T$ thermoelectric materials. Zintl phases are made up of electropositive cations (typically, Group 1 and 2) which donate their electrons to electronegative anions; which, in turn, use them to form bonds in order to satisfy valence. ${ }^{10}$ The structural requirements of Zintl phases are explained by assuming the presence of both ionic and covalent contributions to the bonding picture of the structure. Classical Zintl phases are considered to be valence precise semiconductors. ${ }^{11}$ At the border between classical Zintl phases and normal metallic phases, the semiconducting band gap diminishes and metallic conductivity can result. ${ }^{12}$ Metallic phases at this Zintl border are referred to as polar intermetallics. ${ }^{13}$ The simple Zintl formulism can apply to polar intermetallics, but the difference in electronegativity between the cationic and the anionic units is small, giving rise to homometallic and heterometallic bonding. ${ }^{16-18}$ Thus, the term polar intermetallics is more appropriate when emphasizing that the extent of charge transfer between the cations and anions is significantly less than the ionic model, such as is the case in lithium containing Zintl phases. ${ }^{11}$ Bonding within the polyanionic framework using the Zintl formulism is considered localized, 2center-2-electron bonding, whereas in polar intermetallics charge transfer from the cation to the polyanionic unit is incomplete, and delocalized multicenter bonding is included. ${ }^{16-18}$ The term transition metal Zintl phase is another distinction used in the literature to describe transition metal containing compounds that are isostructural to main group metalloid containing Zintl compounds ${ }^{14-16}$ or, in some cases, new transition metal containing structures where the Zintl formulism provides insight into the electronic structure and bonding. ${ }^{22-26}$

In a Zintl phase the Zintl anions (or transition metal-Zintl ions) provide the "electron-crystal" electronic structure through the covalently bonded network of complex anions or metalloids. Fig. 2 shows several examples of antimony containing Zintl phases: $\mathrm{Ca}_{11} \mathrm{GaSb}_{9},{ }^{17} \mathrm{CaZn}_{2} \mathrm{Sb}_{2},{ }^{18}$ and $\mathrm{BaGa}_{2} \mathrm{Sb}_{2} .{ }^{19}$ These compounds are all semiconductors and the bonding can be described via the Zintl formulism as consisting of electropositive cations and complex anions $\left(\mathrm{Ca}_{11} \mathrm{GaSb}_{9}\right)$, a layered net $\left(\mathrm{CaZn}_{2} \mathrm{Sb}_{2}\right)$ or an open framework or tunnel structure $\left(\mathrm{BaGa}_{2} \mathrm{Sb}_{2}\right) . \mathrm{Ca}_{11} \mathrm{GaSb}_{9}$ consists of $11 \mathrm{Ca}^{2+}$ cations which donate their electrons to $\mathrm{GaSb}_{4}{ }^{9-}$ tetrahedron, $\mathrm{Sb}_{2}{ }^{4-}$ dumbbell, and $3 \mathrm{Sb}^{3-}$ isolated anions. The $\mathrm{Sb}$ anions are considered isolated since there are no near neighbor $\mathrm{Sb}$ atoms that can be considered bonding. These anions are 6 coordinated by the alkaline earth cation. One might expect low lattice thermal conductivity with this complex structure. The $\mathrm{CaZn}_{2} \mathrm{Sb}_{2}$ structure is described in detail below. $\mathrm{BaGa}_{2} \mathrm{Sb}_{2}$ is made up of $\mathrm{Ba}^{2+}$ cations within a $\left(\mathrm{Ga}_{2} \mathrm{Sb}_{2}\right)^{2-}$ network that is composed of $\left(\mathrm{Ga}_{2} \mathrm{Sb}_{6 / 3}\right)^{2-}$ units which can be described as a $\mathrm{Ga}-$ $\mathrm{Ga}$ ethane-like staggered conformation with the $6 \mathrm{Sb}$ atoms. These $\mathrm{Ga}_{2} \mathrm{Sb}_{6 / 3}$ units are linked in a way that leaves holes in the structure as shown in Fig. 2. Such an electron precise compound will naturally form bonding and antibonding bands that will create the necessary semiconducting band-gap. The covalent character of the polyanion bonding can provide high mobility to the charge carriers. Because the band gap arises due to the separation of bonding and antibonding states in the anions, the existence of nonbonding or partially bonding states can fill the gap region with states and make the compound metallic. In contrast, more electronegative elements (such as oxygen and sulfur) will have ionic character to their bonding which will trap carriers and reduce mobility. Zintl anions made from heavier, softer atoms will naturally have lower lattice thermal conductivity. In addition these heavy atoms make smaller band gap semiconductors that are more amenable to the high dopant concentrations necessary for thermoelectrics. The cations in Zintl phases provide regions that can be alloyed or precisely doped to control electron concentration and disrupt phonon transport through alloy scattering. In this way, the thermal and electronic properties of Zintl phases can be tuned to optimize thermoelectric performance. ${ }^{18}$

\section{Zintl phase thermoelectrics}

An example of the flexibility of a Zintl phase with potentially good thermoelectric properties is the solid solution $\mathrm{Ca}_{x} \mathrm{Yb}_{1-x} \mathrm{Zn}_{2} \mathrm{Sb}_{2}$ (Fig. 3). These materials contain electron precise $\mathrm{Zn}-\mathrm{Sb}$ anionic 
A
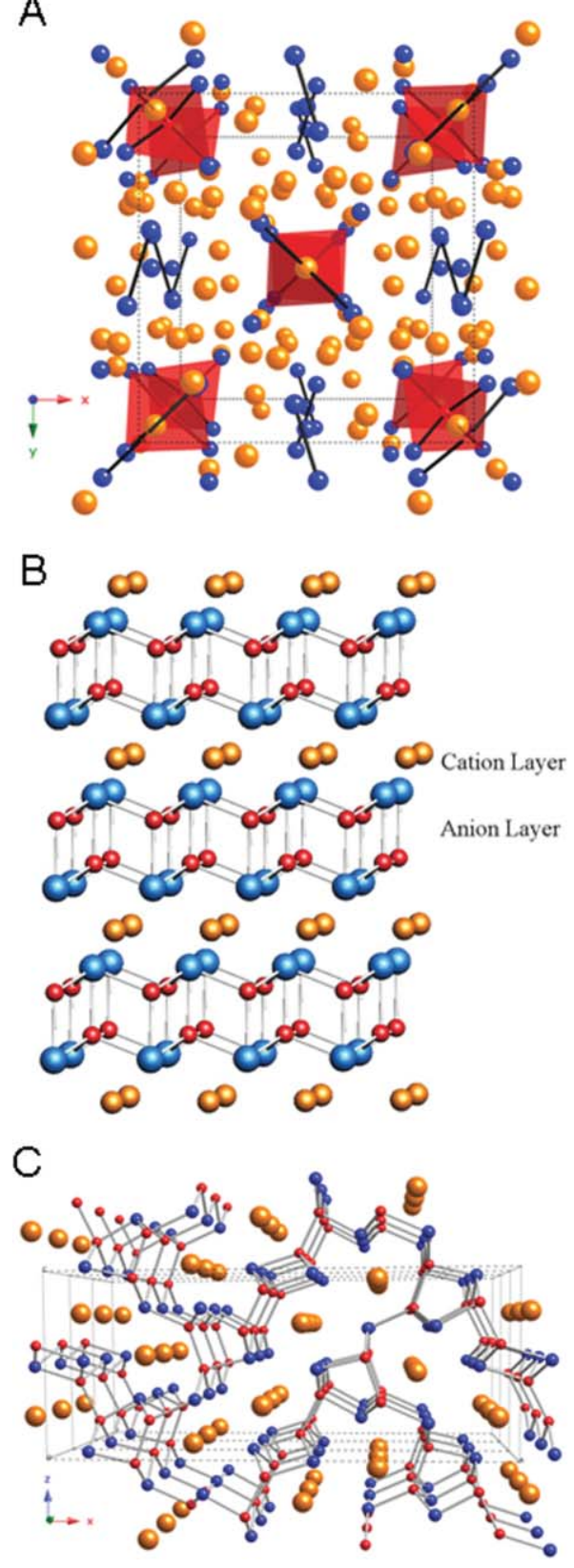

Fig. 2 Example structures of Zintl phases showing the covalently bonding regions (blue and red atoms) separate from the ionic regions (yellow atoms). (A) $\mathrm{Ca}_{11} \mathrm{AlSb}_{9}$, (B) $\mathrm{CaZn}_{2} \mathrm{Sb}_{2}$, and (C) $\mathrm{BaGa}_{2} \mathrm{Sb}_{2}$. The covalent bonded regions provide 'electron-crystal' properties such as high mobility, while the ionic region can be doped or alloyed for tuning of the electron concentration and creating disorder to give 'phonon-glass' properties. The gold, blue and red spheres represent the Zintl cation, antimony, and transition metal atoms, respectively.

sheets. The bonding within the sheets should have mostly covalent character due to the similar electronegativity of $\mathrm{Zn}$ and $\mathrm{Sb}$. Formally, the structure consists of $\mathrm{A}^{2+}$ cations which donate electrons to the $\left(\mathrm{Zn}_{2} \mathrm{Sb}_{2}\right)^{2-}$ framework. The $\left(\mathrm{Zn}_{2} \mathrm{Sb}_{2}\right)^{2-}$ framework has good hole mobility due to its low polarity, in contrast to the polar covalent $\mathrm{Mg}_{3} \mathrm{Sb}_{2}$ with similar structure which despite some promising reports, is a poor thermoelectric. ${ }^{20}$ The bonding to the $\mathrm{Ca}$ site is ionic and $\mathrm{CaZn}_{2} \mathrm{Sb}_{2}$ is a semiconductor with a band

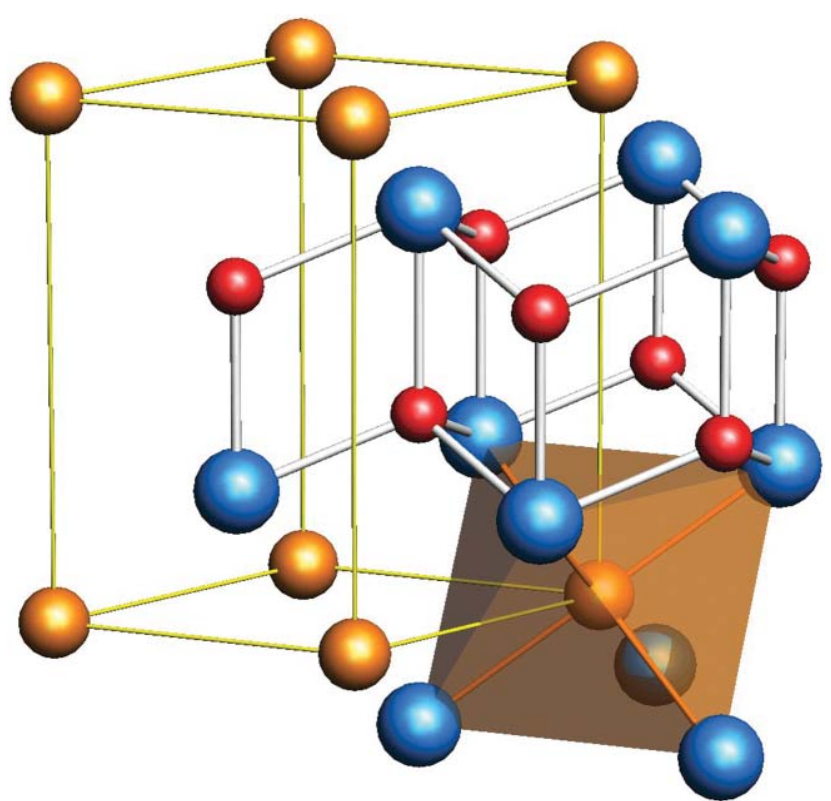

Fig. 3 A view of the structure of $\mathrm{CaZn}_{2} \mathrm{Sb}_{2}$ showing the bonding within the $\mathrm{Zn}-\mathrm{Sb}$ layers between planes of $\mathrm{Ca}^{+2}$. The covalent bonding in the $\mathrm{Zn}_{2} \mathrm{Sb}_{2}{ }^{2-}$ layers provides 'electron-crystal' properties such as high mobility, while substitutions (with $\mathrm{Yb}$ for instance) can be made at the $\mathrm{Ca}$ site for tuning of the electron concentration and creating disorder for 'phonon-glass' properties. The gold, blue and red spheres represent $\mathrm{Ca}$, $\mathrm{Sb}$, and $\mathrm{Zn}$ atoms, respectively.

gap of about $0.25 \mathrm{eV}$. The less electropositive $\mathrm{Yb}$ cation makes $\mathrm{YbZn}_{2} \mathrm{Sb}_{2}$ slightly electron deficient so that it is a heavily doped p-type semiconductor with metal-like transport properties. The complete solid solution $\mathrm{Ca}_{x} \mathrm{Yb}_{1-x} \mathrm{Zn}_{2} \mathrm{Sb}_{2}$ forms and the material transitions from a classical Zintl phase (Ca-rich semiconductor) to a heavily doped polar intermetallic (Yb-rich metal). Aside from carrier concentration, the electronic band structure of the conducting $\mathrm{Zn}_{2} \mathrm{Sb}_{2}$ framework does not change appreciably as evidenced by the invariance of the hole effective mass as a function of $x$ in $\mathrm{Ca}_{x} \mathrm{Yb}_{1-x} \mathrm{Zn}_{2} \mathrm{Sb}_{2}{ }^{18}$ Thus, by alloying isoelectronic species which different donor characteristics on the cationic site, a gradual tuning of the hole concentration is enabled which permits the optimization of $z T$. In addition, the disorder scattering introduced by alloying $\mathrm{Ca}$ and $\mathrm{Yb}$ on the cation site noticeably reduces the lattice component of the thermal conductivity. However, the relatively simple structure prevents the thermal conductivity from being low enough to achieve $z T>1$.

$\mathrm{Zn}_{4} \mathrm{Sb}_{3}$ is another example of a high $z T$ material recently identified as a complex, electron precise $\left(\mathrm{Zn}_{13} \mathrm{Sb}_{10}\right)$ structure ${ }^{21}$ with two types of antimony atoms: $\mathrm{Sb}-\mathrm{Sb}$ bonded dumbbells, $\mathrm{Sb}_{2}{ }^{4-}$, and isolated $\mathrm{Sb}^{3-}$ without bonds to other antimony atoms. In the Zintl formulism, it would be considered to contain $13 \mathrm{Zn}^{2+}$ cations along with $6 \mathrm{Sb}^{3-}$ anions, and $2 \mathrm{Sb}_{2}{ }^{4-}$ dumbbells. However, this ionic description is not reflected in the true electronic structure, which is better described by complex, delocalized multicenter bonding, ${ }^{22}$ which imposes a local structure visible in the low temperature polymorph. The room temperature $\mathrm{Zn}_{4} \mathrm{Sb}_{3}$ phase maintains this delocalized multicenter bonding in the local structure ${ }^{23}$ but at longer length scales (>10 $\AA$ ) the $\mathrm{Zn}$ atoms appear disordered. Thus, in the high-symmetry room temperature structure, $20 \%$ of the $\mathrm{Zn}$ atoms appear in interstitial sites (Fig. 4). The high $z T$ 


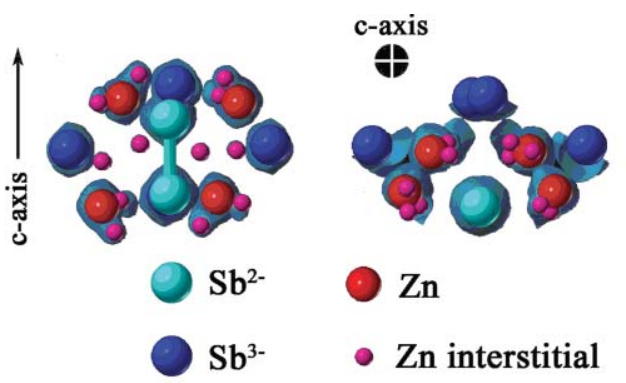

Fig. $4 \mathrm{~A}$ view of the structure of $\mathrm{Zn}_{4} \mathrm{Sb}_{3}$ including an electron density map that clearly shows the existence of interstitial $\mathrm{Zn}$ atoms.

in $\mathrm{Zn}_{4} \mathrm{Sb}_{3}$ can be traced to the low lattice thermal conductivity (less than $1 \mathrm{~W} \mathrm{~m}^{-1} \mathrm{~K}^{-1}$ ) which is attributed to the complex, $\mathrm{Zn}$ interstitial containing structure.

Binary Zintl compounds containing Group 1 or 2 cations can also form complex structures providing phonon-glass qualities. A good example is the $\mathrm{A}_{11} \mathrm{Sb}_{10}$ structure type ${ }^{34-36}$ which can be described by the Zintl formulism as containing $44 \mathrm{~A}^{2+}$ cations with two $\left[\mathrm{Sb}_{4}\right]^{4-}$ squares, eight $\left[\mathrm{Sb}_{2}\right]^{4-}$ dumbbells, and $16 \mathrm{Sb}^{3-}$ anions (Fig. 5) in each unit cell. Indeed this complex structure with multiple anion types has remarkably low lattice thermal conductivity (about $1 \mathrm{~W} \mathrm{~m}^{-1} \mathrm{~K}^{-1}$ ) for such a high melting point compound $(1200 \mathrm{~K}) .^{24}$ Because the electrons are well accounted for in the bonding, the electronic structure is like that of a semiconductor satisfying the electron-crystal requirement. $\mathrm{Ca}_{11} \mathrm{Sb}_{10}$ behaves like a lightly doped semiconductor while $\mathrm{Yb}_{11} \mathrm{Sb}_{10}$ is more metallic like a heavily doped semiconductor. Similar to

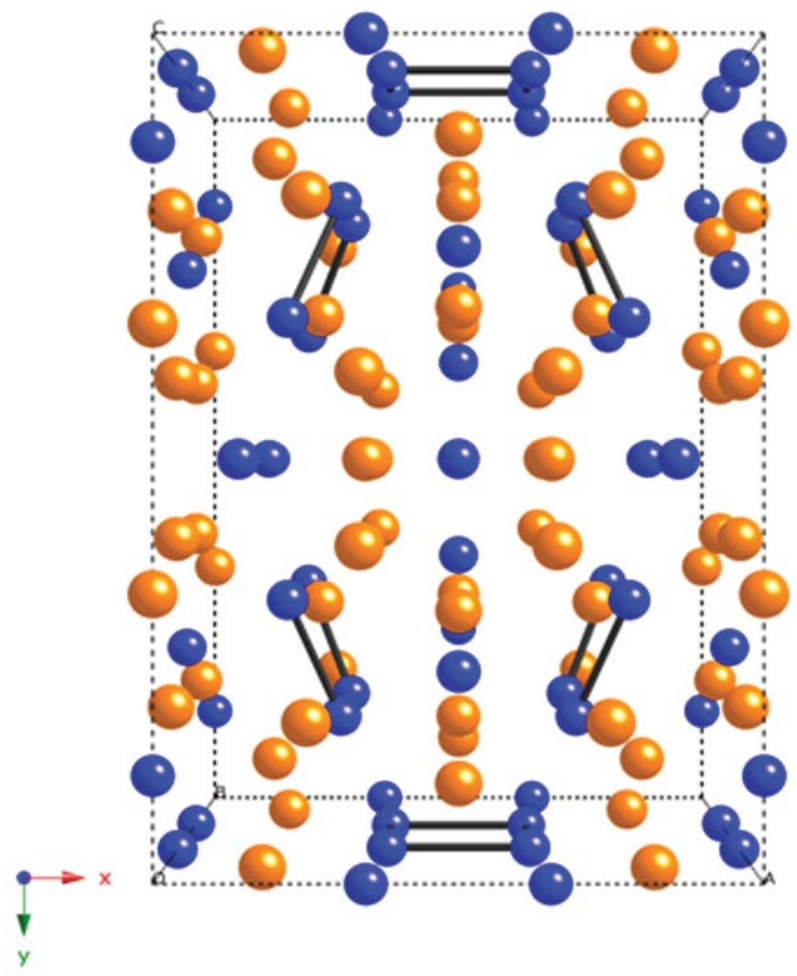

Fig. 5 A view of the structure of $\mathrm{A}_{11} \mathrm{Sb}_{10}$ showing the 3 distinct types of $\mathrm{Sb}$ atoms: $\mathrm{Sb}$ square rings, $\mathrm{Sb}$ dumbbells and isolated $\mathrm{Sb}$ ions. $\mathrm{A}$ and $\mathrm{Sb}$ are designated by gold and blue spheres.
$\mathrm{Yb}_{x} \mathrm{Ca}_{1-x} \mathrm{Zn}_{2} \mathrm{Sb}_{2}$, precise tuning of the semiconductor to metal electronic properties should be possible in $\mathrm{Yb}_{x} \mathrm{Ca}_{11-x} \mathrm{Sb}_{10}$. The $\mathrm{A}_{11} \mathrm{Sb}_{10}$ materials, however, show signs of bipolar conduction (containing both free electrons and holes) which leads to low, compensated thermopower and therefore low $z T$.

Indeed, a number of materials identified as good thermoelectrics contain structures and bonding much more complicated than the simple alloys of traditional thermoelectric materials, and therefore benefit from a Zintl or polar intermetallic description of their structures, such as clathrates, ${ }^{7}$ which are not described in this short perspective. Similar to the example provided above with $\mathrm{CaZn}_{2} \mathrm{Sb}_{2}$, the electron transport properties can be finely tuned and optimized for thermoelectric applications.

\section{Transition metal Zintl phases}

Antimonide skutterudites, ${ }^{25}$ for example, have been found to be good thermoelectric materials with maximum $z T$ in the 0.8 to 1.1 range for both $n$-type and $\mathrm{p}$-type. The skutterudites structure contains a square ring of $\mathrm{Sb}$ atoms at typical $\mathrm{Sb}-\mathrm{Sb}$ bonding distances so that this unit can be considered a $\mathrm{Sb}_{4}{ }^{4-}$ Zintl anion with formal oxidation state of $\mathrm{Sb}^{1-}$. Because of the similar electronegativity with the transition metal (commonly Co or isoelectronic species) the interaction between the antimony and the transition metal will be strongly covalent, producing a high mobility compound semiconductor. The structure allows a number of alloying possibilities on both the transition metal and $\mathrm{Sb}$ sites. In addition, there is a rather large empty void in the skutterudite that will readily accept a large alkaline earth or rare earth cation, making what is known as a filled skutterudite (shown in Fig. 6). However, solubility of alloys and filling atoms is limited to a narrow range of compositions nearly isoelectronic to $\mathrm{CoSb}_{3}$ (within about $1 / 4 \mathrm{e}^{-}$per transition metal). Thus, the materials

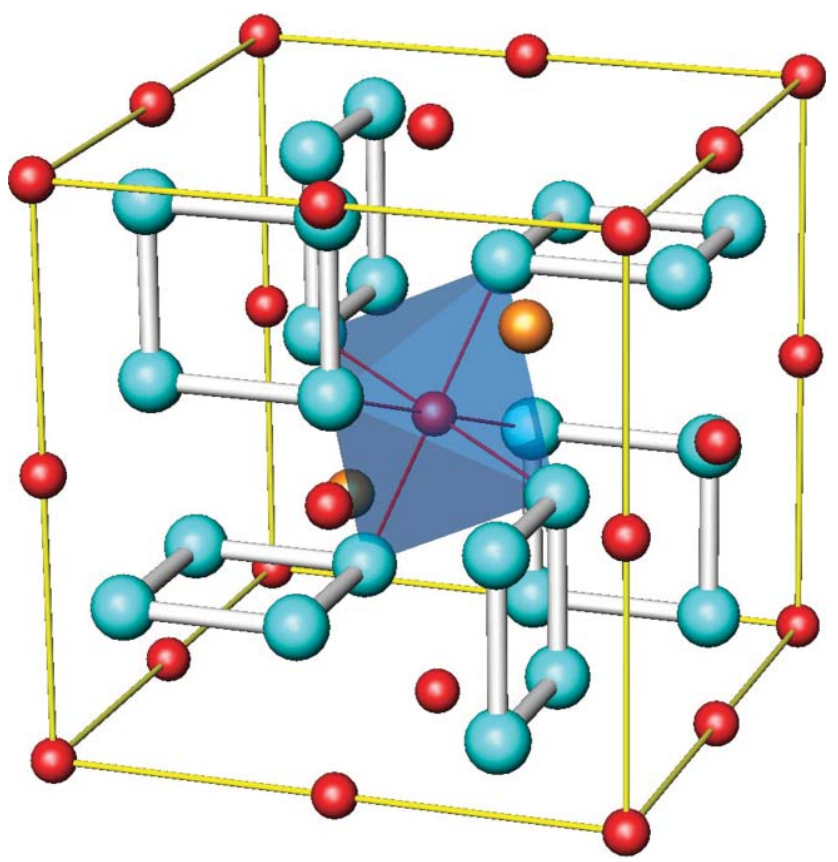

Fig. 6 A view of the structure of the filled skutterudite emphasizing the $\mathrm{Sb}_{4}{ }^{4-}$ square ring and the metal centered octahedron. The gold, blue, and red spheres represent the cation, antimonide, and metal atoms, respectively. 
are nearly electron precise qualifying the filled compositions as transition metal Zintl phases. The disorder and 'rattling' of the filling atom has been associated with a reduction in lattice thermal conductivity and is another example (in addition to $\mathrm{AZ} \mathrm{n}_{2} \mathrm{Sb}_{2}$ described above) of the ability of Zintl phases to separate electroncrystal regions from phonon-glass regions.

Materials crystallizing in the $\mathrm{Ir}_{3} \mathrm{Ge}_{7}$ structure, such as $\mathrm{Mo}_{3} \mathrm{Sb}_{7}$ (shown in Fig. 7), have also been shown to be promising for thermoelectric applications. $\mathrm{Mo}_{3} \mathrm{Sb}_{7}$, like the antimonide skutterudites, is capable of achieving a low lattice thermal conductivity and good Seebeck coefficient, resulting in a good figure of merit. $\mathrm{Mo}_{3} \mathrm{Sb}_{7}$ is a complex three-dimensional arrangement of atoms comprised of antimony dimers, square antiprisms and empty $\mathrm{Sb}_{8}$ cubes. ${ }^{26,27}$ The $\mathrm{Sb}_{8}$ cubes have the potential to house interstitial elements, opening opportunities for the addition of dopants. In addition, the structure of $\mathrm{Mo}_{3} \mathrm{Sb}_{7}$ is reported to be able to accommodate a number of different valence-electron-counts (51 to 56) and elemental constituents possibly further aiding in the optimization of its thermoelectric properties. If one counts only the short $\mathrm{Sb}-\mathrm{Sb}$ dimer distance as a bond (these bonds are emphasized in Fig. 7), the valence of $\mathrm{Mo}_{3} \mathrm{Sb}_{7}$ can be rationalized as being two electrons short of valence balance $\left(\mathrm{Mo}_{3}^{5+} \mathrm{Sb}_{4}^{2-} \mathrm{Sb}_{3}^{3-}\right)$. Substitution of $\mathrm{Te}$ for $\mathrm{Sb}$ will add an electron and bring the system closer to valence balance. This accounts for the p-type, nearly semiconducting behavior of $\mathrm{Mo}_{3} \mathrm{Sb}_{7-x} \mathrm{Te}_{x}$ as $x$ approaches 2. For example, the addition of $\mathrm{Te}(x=1.6)$ into $\mathrm{Mo}_{3} \mathrm{Sb}_{7-x} \mathrm{Te}_{x}$ increases the Seebeck coefficient and decrease the thermal conductivity. The combination of resistivity, Seebeck coefficient and thermal conductivity results in a maximum $z T$ of $\sim 0.8$ at $1050 \mathrm{~K}^{26}$ Much efforts are in progress to tune this material further, with attention being focused on modifying the carrier concentration (a.k.a. controlling of the valence-electron-count) and filling the $\mathrm{Sb}_{8}$ cube voids. $\mathrm{Mo}_{3} \mathrm{Sb}_{7}$ shows yet another example of a complex transition metal Zintl phase capable of achieving an extremely reduced lattice thermal conductivity and good thermoelectric properties. However, even though the reduced lattice thermal conductivity of $\mathrm{Mo}_{3} \mathrm{Sb}_{7}$ is good for thermoelectric applications,

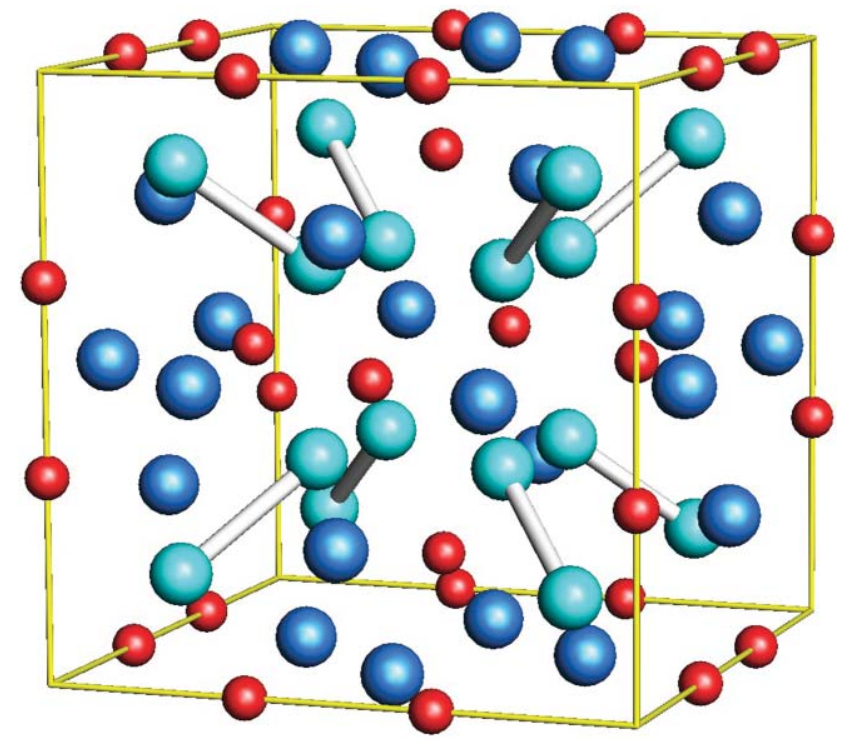

Fig. 7 A view of the $\mathrm{Mo}_{3} \mathrm{Sb}_{7}$ structure where the $\mathrm{Sb}-\mathrm{Sb}$ bonds (light blue) and isolated $\mathrm{Sb}$ (dark blue) and Mo (red) are shown. the recently published thermoelectric data for $\mathrm{Yb}_{14} \mathrm{MnSb}_{11}$ reveals an even lower lattice thermal conductivity.

The recent discovery of high $z T$ in $\mathrm{Yb}_{14} \mathrm{MnSb}_{11}$ demonstrates the successful combination of electron-crystal phonon-glass properties afforded by Zintl phases. ${ }^{8} \mathrm{Yb}_{14} \mathrm{MnSb}_{11}$ is composed of distinct anionic units (like $\mathrm{Yb}_{11} \mathrm{Sb}_{10}$ ) rather than the extended structures shown in Fig. 2 (such as $\mathrm{CaZn}_{2} \mathrm{Sb}_{2}$ ). The structure consists of (Fig. 8) $\left[\mathrm{MnSb}_{4}\right]^{9-}$ tetrahedra, polyatomic $\left[\mathrm{Sb}_{3}\right]^{7-}$ anions, as well as isolated $\mathrm{Sb}^{3-}$ anions, where the electrons are donated by the $\mathrm{Yb}$, to give $\mathrm{Yb}^{2+}$ cations. This compound is isostructural to the well-known Zintl phase, $\mathrm{Ca}_{14} \mathrm{AlSb}_{11}{ }^{28}$ with ionic bonds between the cation and the anionic units. In principle, since these anionic units are ionically bonded together through cations, a high temperature melting point is also expected, providing incentive for high temperature stable thermoelectric properties. While this structure is clearly much more complex than the example provided above for $\mathrm{CaZn}_{2} \mathrm{Sb}_{2}$, it has many of the same attributes and provides for the possibilities of fine tuning.

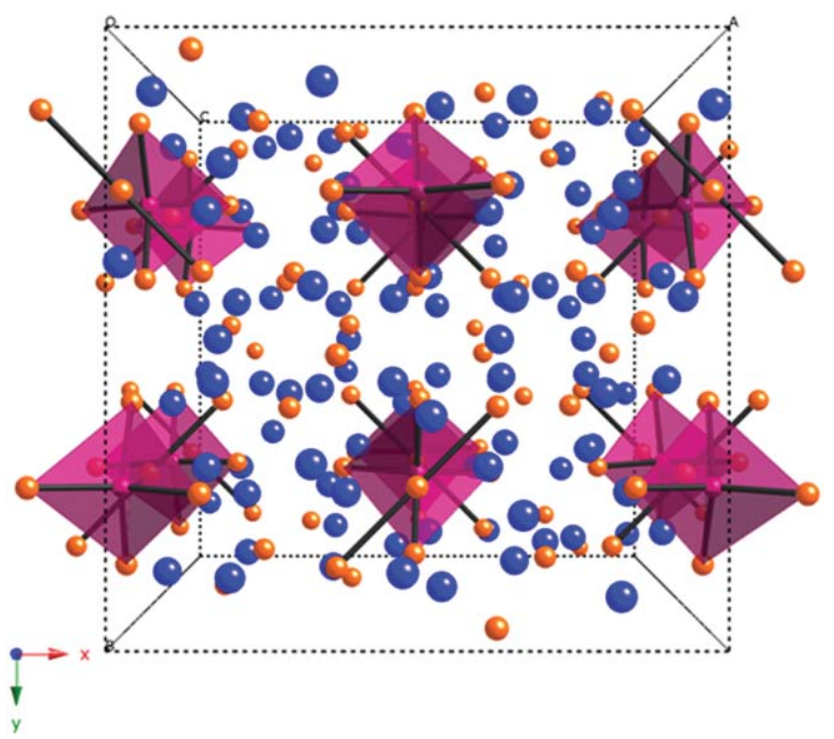

Fig. 8 A view of $\mathrm{A}_{14} \mathrm{MPn}_{11}$ structure $(\mathrm{A}=2+$ cation, $\mathrm{M}=$ metal, $\mathrm{Pn}=\mathrm{P}$, $\mathrm{As}, \mathrm{Sb}, \mathrm{Bi}$ ), where the $\mathrm{MPn}_{4}$ polyhedra and $\mathrm{Pn}_{3}$ unit are shown. The gold, blue, and red spheres represent the $\mathrm{A}, \mathrm{Pn}$, and $\mathrm{M}$ atoms, respectively.

The generic expression for this structure type is $\mathrm{A}_{14} \mathrm{MPn}_{11}$, where $A$ is a heavy or alkaline earth metal, $\mathrm{M}$ is a transition or main group metal, and $\mathrm{Pn}$ is a heavier Group 15 element ( $\mathrm{P}, \mathrm{As}, \mathrm{Sb}$, or $\mathrm{Bi}) .{ }^{10,16}$ The electronic structure of the main group analog is consistent with the Zintl formulism. ${ }^{29}$ The various sites allow for the potential tuning of the electronic and thermal properties through elemental substitutions. Substituting on the cationic metal site, A, allows for possible tuning of the carrier concentration and disorder scattering of phonons, while substituting on the metal site, $\mathrm{M}$, enables possible tuning of the electronic parameters.

$\mathrm{Yb}_{14} \mathrm{MnSb}_{11}$ is best considered as a polar intermetallic (because of the metallic transport properties) $)^{30,31}$ compared with the transition metal Zintl phase, $\mathrm{Ca}_{14} \mathrm{MnSb}_{11}$ (semiconducting). ${ }^{32}$ However, the presence of the transition metal changes the electronic structure and bonding within this structure type. ${ }^{33} \mathrm{X}$-Ray magnetic circular dichroism (XMCD) $)^{34}$ and $\mathrm{XPS}^{35}$ measurements show that, indeed, the ytterbium is in the $\mathrm{Yb}^{2+}$ valence state replacing $\mathrm{Ca}^{2+}$; 


\section{A}

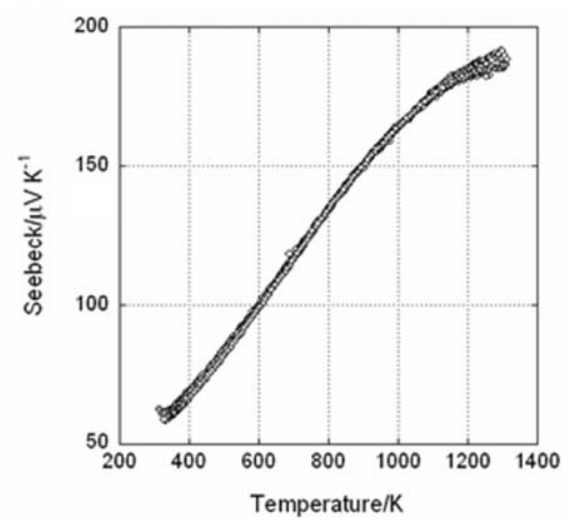

B

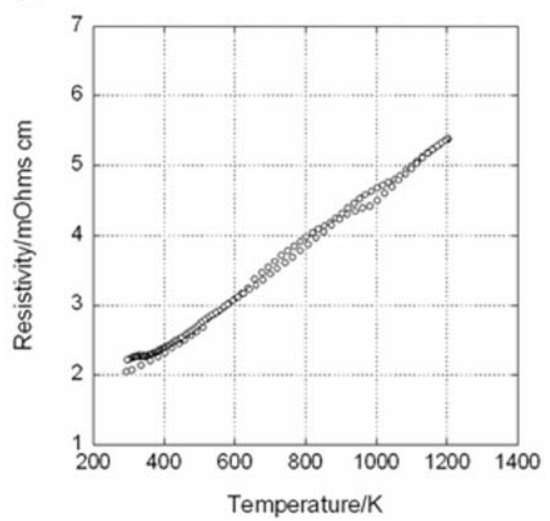

Fig. 9 (A) Seebeck and (B) resisitivity vs. temperature for $\mathrm{Yb}_{14} \mathrm{MnSb}_{11}$.

however, the manganese has been shown to be in the $\mathrm{Mn}^{2+}$ valence state, supplying one less electron than $\mathrm{Al}^{3+}$. This shortage of electrons $\left(1.3 \times 10^{21}\right.$ holes $\left.\mathrm{cm}^{-3}\right)$ explains the material's $\mathrm{p}$-type behavior and corresponds well to the measured carrier concentration by Hall effect. This high carrier concentration indicates that the hole mobility is not particularly high, only about $3 \mathrm{~cm}^{2} \mathrm{~V}^{-1} \mathrm{~s}^{-1}$, but the high thermopower implies a large effective mass $m^{*} \sim 3 m_{\mathrm{e}}$ using eqn (1). Fig. 9 shows the temperature dependent Seebeck coefficient and resistivity. The Seebeck coefficient is positive and increases with increasing temperature. The resistivity increases with increasing temperature and reaches a maximum at about $5 \mathrm{~m} \Omega \mathrm{cm}$ at $1200 \mathrm{~K}$. These measurements also show that this is a stable high temperature material, with no melting or structural transition up to $1200 \mathrm{~K}$. A reduction in carrier concentration, by substitution of $\mathrm{La}^{3+}$ for $\mathrm{Yb}^{2+}$ on the A site or by replacing $\mathrm{Mn}^{2+}$ with $\mathrm{Al}^{3+}$ should bring the peak in $z T$ to lower temperatures, providing higher average $z T$ over a wider temperature range. In addition, isoelectronic alloying on the A site, for example by replacing some $\mathrm{Yb}^{2+}$ with $\mathrm{Ca}^{2+}$ should result in a further reduction in lattice thermal conductivity as seen in $\mathrm{Yb}_{1-x} \mathrm{Ca}_{x} \mathrm{Zn}_{2} \mathrm{Sb}_{2} .{ }^{18}$

The total thermal conductivity of $\mathrm{Yb}_{14} \mathrm{MnSb}_{11}$, shown in Fig. 10, is remarkably low ranging between $\sim 0.7-0.9 \mathrm{~W} \mathrm{~m}^{-1} \mathrm{~K}^{-1}$ for the temperatures of 300-1275 K. Subtracting the electronic contribution results in an extremely low lattice thermal conductivity. This

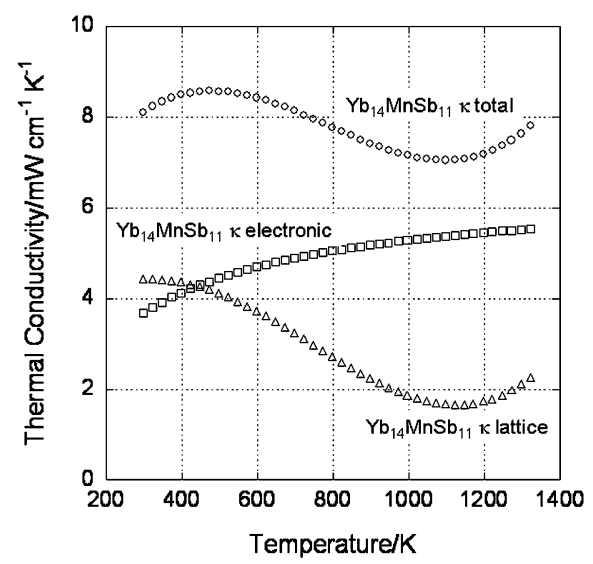

Fig. 10 Thermal conductivity $v$ s. temperature for $\mathrm{Yb}_{14} \mathrm{MnSb}_{11}$. Total thermal conductivity, electronic thermal conductivity, and lattice thermal conductivity are shown.

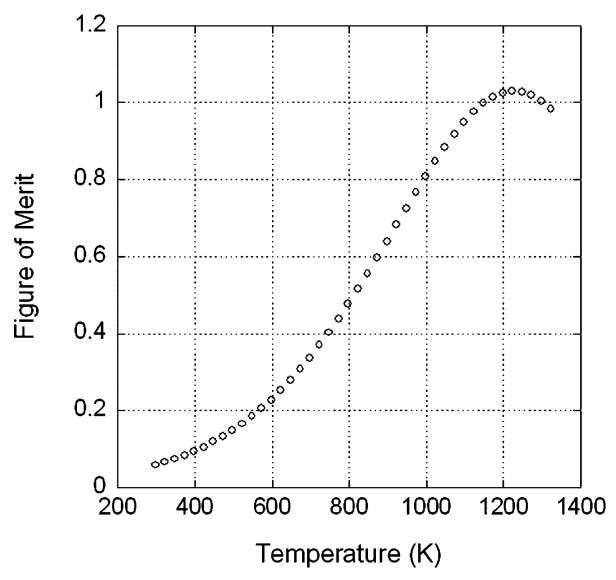

Fig. $11 z T$, Figure of merit, vs. temperature for $\mathrm{Yb}_{14} \mathrm{MnSb}_{11}$.

thermal conductivity is comparable to that of a glass,$^{36}$ heavily contributing to the material's high figure of merit (Fig. 11). The low values can be attributed to the complexity of the structure (limiting the phonon mean-free path) and heavy atomic mass (reducing the fraction of atomic vibrational modes that carry heat efficiently) of the crystal.

$\mathrm{Yb}_{14} \mathrm{MnSb}_{11}$ possesses a high figure of merit $(z T \geq 1)$ in the temperature region (up to $1275 \mathrm{~K}$ ) where the state-of-theart material is $\mathrm{p}-\mathrm{Si}_{1-x} \mathrm{Ge}_{x}$ alloy ( $\mathrm{SiGe}$ ), with a maximum $z T=$ $0.6{ }^{37,38}$ Fig. 12 compares the most competitive p-type materials, showing the improvement of $\mathrm{Yb}_{14} \mathrm{MnSb}_{11}$ in the high temperature range. For highest efficiency $\mathrm{Yb}_{14} \mathrm{MnSb}_{11}$ would be most effectively utilized in a segmented thermoelectric device where other materials with high $z T$ at lower temperatures such as TAGS are used in the lower temperature region. ${ }^{39}$ In order to utilize a segmented element, a thermoelectric compatibility factor, $s$, must also be considered. The compatibility factor takes into account the requirement that the same electrical current and similar heat must flow through each segment. Fig. 13 shows the compatibility factor, $s$, for materials being considered for such a device. If the compatibility factors differ by more than a factor of 2 , efficiency of the device is substantially reduced. $\mathrm{Yb}_{14} \mathrm{MnSb}_{11}$ is much more compatible than the current high temperature p-type material, $\mathrm{SiGe}$, for segmentation with lower temperature high $z T$ materials such as TAGS because of the closer compatibility factor. 


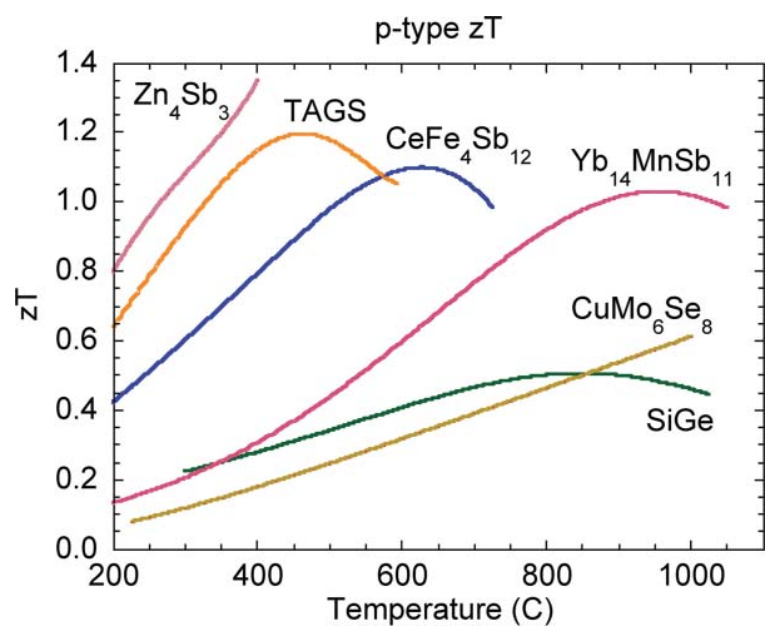

Fig. 12 Figure of merit as a function of temperature for a number of p-type materials (TAGS: $\left.(\mathrm{GeTe})_{0.85}\left(\mathrm{AgSbTe}_{2}\right)_{0.15}\right)$. Reprinted with permission from Chem. Mater., 2006, 18, 1873. (C) American Chemical Society 2006.

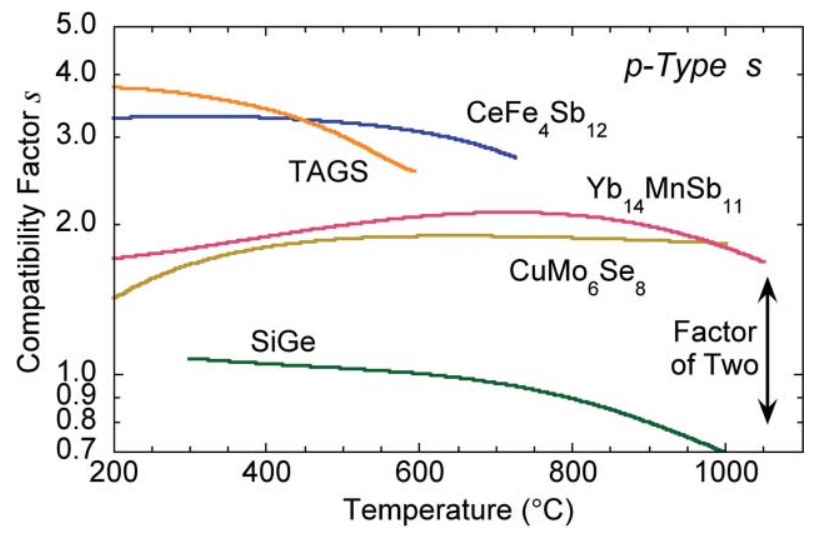

Fig. 13 Thermoelectric compatibility factor, $s$, for a variety of p-type materials. $\mathrm{Yb}_{14} \mathrm{MnSb}_{11}$ compatibility is improved by a factor of 2 over the current material, SiGe. (TAGS: $\left.(\mathrm{GeTe})_{0.85}\left(\mathrm{AgSbTe}_{2}\right)_{0.15}\right)$. Reprinted with permission from Chem. Mater., 2006, 18, 1873. (C) American Chemical Society 2006.

In addition, because of the many known compositions with the same structure type, it also introduces a chemically rich, bulk material into the thermoelectric community that can be further optimized.

\section{Conclusions and outlook}

Zintl phases possess the structural characteristics needed for electron-crystal phonon-glass properties that lead to high thermoelectric efficiency $(z T)$. They combine distinct regions of covalent bonding ideal for electron-crystal properties with ionically bonded cations that can be easily substituted for precise tuning of electronic properties and introducing disorder. The complex structures and disorder creates phonon-glass properties resulting in low lattice thermal conductivity, essential for high $z T$. A good understanding of the interaction of the bonding and chemistry with the transport properties in these compounds leads to a focused search for new materials and a rational approach to optimizing the electronic and thermal properties.

\section{Acknowledgements}

Our work covered in this Perspective has been generously supported by the National Science Foundation (DMR-0600742) and NASA-Jet Propulsion Laboratory.

\section{References}

1 CRC Handbook of Thermoelectrics, ed. D. M. Rowe, CRC Press, New York, 1995.

2 G. A. Slack, in CRC Handbook of Thermoelectrics, ed. D. M. Rowe, CRC Press, New York, 1995, p. 407-440.

3 M. Cutler, J. F. Leavy and R. L. Fitzpatrick, Electronic Transport in Semimetallic Cerium Sulfide, Phys. Rev., 1964, 133, 1143-\&.

4 K. Koumoto, I. Terasaki and R. Funahashi, Complex oxide materials for potential thermoelectric applications, MRS Bull., 2006, 21, 206210 .

5 H. Böttner, G. Chen and R. Venkatasubramanian, Aspects of ThinFilm Superlattice Thermoelectric Materials, Devices, and Applications, MRS Bull., 2006, 31, 211-217.

6 A. M. Rao, X. Ji and T. M. Tritt, Properties of Nanostructured OneDimensional and Composite Thermoelectric Materials, MRS Bull., 2006, 31, 218-223.

7 G. S. Nolas, J. Poon and M. Kanatzidis, Recent Developments in Bulk Thermoelectric Materials, MRS Bull., 2006, 31, 199-205.

8 S. R. Brown, S. M. Kauzlarich, F. Gascoin and G. J. Snyder, Yb14MnSb11. New high efficiency thermoelectric material for power generation, Chem. Mater., 2006, 18, 1873-1877.

9 W. M. Yim and F. D. Rosi, Compound tellurides and their alloys for Peltier cooling. Review, Solid-State Electron., 1972, 15, 1121-1140.

10 Chemistry, Structure, and Bonding of Zintl Phases and Ions, ed. S. M. Kauzlarich, VCH Publishers, Inc, New York, 1996.

11 R. Nesper, Structure and Chemical bonding in Zintl-phases containing Li, Prog. Solid State Chem., 1990, $20,1$.

12 G. J. Miller, in Chemistry, Structure, and Bonding of Zintl Phases and Ions, ed. S. M. Kauzlarich, VCH, New York, 1996, p. 1-59.

13 C. Lupu, C. Downie, A. M. Guloy, T. A. Albright and J.-G. Mao, $\mathrm{Li}_{17} \mathrm{Ag}_{3} \mathrm{Sn}_{6}$ : A Polar Intermetallic $\pi$-System with Carbonate-like $[\mathrm{AgSn}]^{11-}$ Anions and Trefoil Aromatic $\left[\mathrm{Ag}_{2} \mathrm{Sn}_{3}\right]^{6-}$ Layers, J. Am. Chem. Soc., 2004, 126, 4386-4397.

14 S. M. Kauzlarich, A Rational Approach to Solid State Synthesis-The Zintl Concept, Comments Inorg. Chem., 1990, 10, 75.

15 S. M. Kauzlarich, in Chemistry, Structure, and Bonding of Zintl Phases and Ions, ed. S. M. Kauzlarich, VCH Publishers, Inc., New York, 1996, pp. $245-274$.

16 S. M. Kauzlarich, A. C. Payne and D. J. Webb, in Magnetism: Molecules to Materials III, ed. J. S. Miller and M. Drillon, Wiley-VCH, Weinham, 2002, pp. 37-62.

17 D. M. Young and S. M. Kauzlarich, Preparation, Structure, and Electronic Properties of $\mathrm{Ca}_{11} \mathrm{MSb}_{9}(\mathrm{M}=\mathrm{Al}, \mathrm{Ga}, \mathrm{In})$, Chem. Mater., 1995, 7, 206-209.

18 F. Gascoin, S. Ottensmann, D. Stark, S. M. Haile and G. J. Snyder, Zintl phases as thermoelectric materials: Tuned transport properties of the compounds $\mathrm{Ca}_{x} \mathrm{Yb}_{1-x} \mathrm{Zn}_{2} \mathrm{Sb}_{2}$, Adv. Funct. Mater., 2005, 15, 1860-1864.

19 S. J. Kim and M. G. Kanatzidis, A unique framework in $\mathrm{BaGa}_{2} \mathrm{Sb}_{2}$ : A new Zintl phase with large tunnels, Inorg. Chem., 2001, 40, 3781-3785.

20 C. L. Condron, S. M. Kauzlarich, F. Gascoin and G. J. Snyder, Thermoelectric properties and microstructure of $\mathrm{Mg}_{3} \mathrm{Sb}_{2}$, J. Solid State Chem., 2006, 179, 2252-2257.

21 G. J. Snyder, M. Christensen, E. Nishibori, T. Caillat and B. B. Iversen, Disordered zinc in $\mathrm{Zn}_{4} \mathrm{Sb}_{3}$ with phonon-glass and electron-crystal thermoelectric properties, Nat. Mater., 2004, 3, 458-463.

22 J. Nylén, M. Andersson, S. Lidin and U. Häussermann, The structure of $\alpha-\mathrm{Zn}_{4} \mathrm{Sb}_{3}$ : Ordering of the Phonon-Glass Thermoelectric Material $\beta-\mathrm{Zn}_{4} \mathrm{Sb}_{3}$, J. Am. Chem. Soc., 2004, 126, 16306-16307.

23 H. J. Kim, E. S. Bözin, S. M. Haille, G. J. Snyder and S. J. L. Billinge, Nano-scale-structural domains in the phonon-glass thermoelectric material, $\mathrm{Zn}_{4} \mathrm{Sb}_{3}$, Phys. Rev. B: Condens. Matter, 2007, B75, 134103.

24 S. R. Brown, S. M. Kauzlarich, F. Gascoin and G. J. Snyder, HighTemperature Thermoelectric Studies of $\mathrm{A}_{11} \mathrm{Sb}_{10}(\mathrm{~A}=\mathrm{Yb}, \mathrm{Ca}) J$ Solid State Chem., 2007, 10.1016/j.jssc.2007.02.007.

25 C. Uher, in Semiconductors and Semimetals, ed. T. M. Tritt, Academic Press,San Diego, 2001, vol. 69, pp. 139-253. 
26 F. Gascoin, J. Rasmussen and G. J. Snyder, High temperature thermoelectric properties of $\mathrm{Mo}_{3} \mathrm{Sb}_{7-x} \mathrm{Te}_{x}$ for $x=1.6$ and 1.5, $J$ Alloys Compd., 2007, 427, 324-329.

27 E. Dashjav, A. Szczepenowska and H. Kleinke, Optimization of the thermopower of the antimonide $\mathrm{Mo}_{3} \mathrm{Sb}_{7}$ by a partial $\mathrm{Sb} / \mathrm{Te}$ substitution, J. Mater. Chem., 2002, 12, 345-349.

28 G. Cordier, H. Schäfer and M. Stelter, Darstellung und Struktur der Verbindung $\mathrm{Ca}_{14} \mathrm{AlSb}_{11}$, Z. Anorg. Allg. Chem., 1984, 519, 183-188.

29 R. F. Gallup, C. Y. Fong and S. M. Kauzlarich, Bonding properties of $\mathrm{Ca}_{14} \mathrm{GaAs}_{11}-\mathrm{a}$ compound containing discrete $\mathrm{GaAs}_{4}$ tetrahedra and a hypervalent $\mathrm{As}_{3}$ polyatomic unit, Inorg. Chem., 1992, 31, 115-118.

30 I. R. Fisher, T. A. Wiener, S. L. Bud'ko, P. C. Canfield, J. Y. Chan and S. M. Kauzlarich, Thermodynamic and transport properties of single-crystal $\mathrm{Yb}_{14} \mathrm{MnSb}_{11}$, Phys. Rev. B: Condens. Matter, 1999, 59, 13829-13834.

31 J. Y. Chan, M. M. Olmstead, S. M. Kauzlarich and D. J. Webb, Structure and Ferromagnetism of the Rare-Earth Zintl Compounds: $\mathrm{Yb}_{14} \mathrm{MnSb}_{11}$ and $\mathrm{Yb}_{14} \mathrm{MnBi}_{11}$, Chem. Mater., 1998, 10, 3583-3588.

32 A. Rehr, T. Y. Kuromoto, S. M. Kauzlarich, J. Delcastillo and D. J. Webb, Structure and Properties of the Transition-Metal Zintl Compounds $\mathrm{A}_{14} \mathrm{MnPn}_{11}(\mathrm{~A}=\mathrm{Ca}, \mathrm{Sr}, \mathrm{Ba} ; \mathrm{Pn}=\mathrm{As}, \mathrm{Sb})$, Chem. Mater. 1994, 6, 93-99.
33 D. Sánchez-Portal, R. M. Martin, S. M. Kauzlarich and W. E. Pickett, Bonding, Moment Formation, and Magnetic Interactions in $\mathrm{Ca}_{14} \mathrm{MnBi}_{11}$ and $\mathrm{Ba}_{14} \mathrm{MnBi}_{11}$, Phys. Rev. B: Condens. Matter, 2002, 65, 144414.

34 A. P. Holm, S. M. Kauzlarich, S. A. Morton, G. D. Waddill, W. E. Pickett and J. G. Tobin, XMCD Characterization of the Ferromagnetic State of $\mathrm{Yb}_{14} \mathrm{MnSb}_{11}$, J. Am. Chem. Soc., 2002, 124, 9894 9898.

35 A. P. Holm, T. C. Ozawa, S. M. Kauzlarich, S. A. Morton, G. D. Waddill and J. G. Tobin, X-ray photoelectron spectroscopy studies of $\mathrm{Yb}_{14} \mathrm{MnSb}_{11}$ and $\mathrm{Yb}_{14} \mathrm{ZnSb}_{11}$, J. Solid State Chem., 2005, 178, 262 269.

36 B. C. Sales, Novel thermoelectric materials, Curr. Opin. Solid State Mater. Sci., 1997, 2, 284-289.

37 C. Wood, High-temperature thermoelectric energy conversionII. Materials survey, Energy Convers. Manage., 1984, 24, 331-343.

38 O. Yamashita and N. Sadatomi, Thermoelectric properties of $\mathrm{Si}_{1-x} \mathrm{Ge}_{x}$ $(x=0.10)$ with alloy and dopant segregations, J. Appl. Phys., 2000, 88, 245-251.

39 G. J. Snyder, Application of the compatibility factor to the design of segmented and cascaded thermoelectric generators, Appl. Phys. Lett., 2004, 84, 2436-2438. 